\title{
Exotic structures in exotic nuclei
}

\author{
F. Miguel Marqués * \\ LPC Caen, Normandie Université, CNRS/IN2P3, ENSICAEN, Caen, France \\ $\star$ marques@lpccaen.in2p3.fr
}

for the SAMURAI Day-One, S18 and S34 collaborations

盟发到

Proceedings for the 24th edition of European Few Body Conference,

Surrey, UK, 2-6 September 2019

doi:10.21468/SciPostPhysProc.3

\begin{abstract}
Nuclei with proton and neutron numbers away from stability are known as exotic, and provide stringent tests of nuclear models, mainly developed for the description of stable nuclei. However, only the most extreme combinations lead to literally exotic structures, with sometimes unexpected properties. In this contribution we review some selected examples around the neutron dripline of very light elements: neutron halo structures with embedded substructures in the boron chain; the emission of neutron pairs in ${ }^{16} \mathrm{Be}$; and the existence of multi-neutron resonances in 'superheavy' hydrogen isotopes.
\end{abstract}

(c) (1) Copyright F. M. Marqués et al.

This work is licensed under the Creative Commons

Attribution 4.0 International License.

Published by the SciPost Foundation.
Received 23-10-2019

Accepted 29-11-2019

Published 24-02-2020

doi:10.21468/SciPostPhysProc.3.001

\section{Introduction}

The composition of the nucleus is relatively simple: an ensemble, more or less balanced, of protons and neutrons. Its structure, however, is well understood only for a finite range of combinations close to the stable equilibrium values, along the so-called valley of stability.

The basic question of what the properties of a system with a given number $Z$ of protons and $N$ of neutrons would be, is still open for most of those numbers. Different nuclear models have been developed based mostly on the description of stable nuclei, and their extrapolations towards unknown $(N, Z)$ combinations usually diverge among themselves. Therefore, the ability to produce and study in the laboratory increasingly exotic combinations has been very valuable in guiding and/or discarding those models.

Ideally, producing the whole range of possible $N$ values for each given element $Z$ would be the ultimate goal. However, when moving up in $Z$ this range becomes increasingly wide specially on the neutron-rich side, due to the need to counterbalance the Coulomb repulsion among the protons and to the absence of a Coulomb barrier for the neutrons.

As such, the most neutron-rich combinations soon become unreachable in the laboratory, making the knowledge of those systems rely exclusively on the ability of nuclear models to predict the properties of any system. 


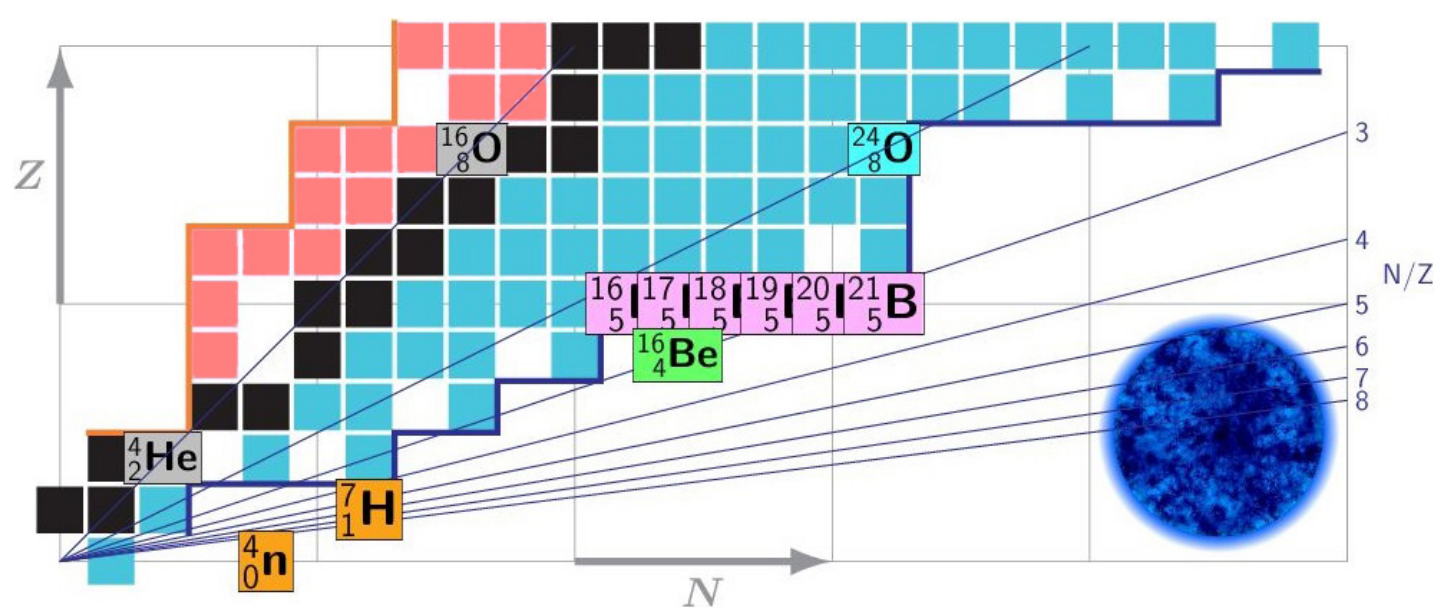

Figure 1: The $(N, Z)$ chart of isotopes up to neon $(Z=10)$. Stable nuclei are represented by black boxes, and exotic nuclei by red (neutron deficient) and cyan (neutron rich) boxes. The exotic structures discussed in this contribution are highlighted in magenta (boron chain), green (dineutron emission) and orange (multi-neutron systems). For comparison, a neutron star has been placed along the $N / Z=8$ line.

On one hand, reaching the $(N, Z)$ limits in the region where they are attainable, presently up to $Z \sim 9$ [1], provides the most stringent test of those models.

On the other hand, even if all combinations away from stability are called exotic, only around the extreme limits do the nuclear properties deviate from smooth trends and generate literally exotic structures, even more challenging for the models.

In this contribution we will review some of these "exotic structures in exotic nuclei", all of them probed at the Radioactive Isotope Beam Factory (RIBF) of the RIKEN Nishina Center, in Japan. They are displayed in Fig. 1, where we see how they spread over $N / Z$ ratios from 2-3 to infinity, in the case of multi-neutron systems.

\section{$2 \mathrm{Z}=5$ : the boron 'matryoshka'}

In the naive shell-model picture, neutron numbers between 8 and 20 correspond to the filling of the $s d$-shell neutron single-particle orbitals $\left(\nu 0 d_{5 / 2}, v 1 s_{1 / 2}, v 0 d_{3 / 2}\right)$. Approaching the neutron dripline, the energies of these orbitals evolve, leading for example to the disappearance of the $N=20$ magic number for $Z=10-12$ and to the appearance of new shell closures at $N=14$ and 16 in the oxygen isotopes [2-4]. These changes dictate the structure and location of the neutron dripline from boron to fluorine, leading for example to the sudden increase of the dripline from $N=16$ to 22 (Fig. 1), known as the "oxygen anomaly" [5].

A more general and fundamental anomaly arises along the boron isotopic chain, in which most of the isotopes lie around the dripline. The weak binding characteristic of the dripline together with clustering phenomena lead to a number of exotic structures: from the proton halo of ${ }^{8} \mathrm{~B}$ [6], through the unbound threshold states of ${ }^{16,18} \mathrm{~B}[7,8]$, to the two-neutron halo of ${ }^{17} \mathrm{~B}$ and the two/four neutron halo/skin of ${ }^{19} \mathrm{~B}$ [9]. More recently, resonances in ${ }^{20,21} \mathrm{~B}$ have been observed [10].

On Fig. 2 we have sketched the complex exotic structures that can be found when going from the bound isotope ${ }^{15} \mathrm{~B}$ up to the resonances in ${ }^{21} \mathrm{~B}$. The first neutron-rich unbound isotope, ${ }^{16} \mathrm{~B}$, exhibits a very narrow, low-energy neutron resonance at about $40 \mathrm{keV}$, that has been measured with a precision of only a few keV. This system of 16 nucleons can be modeled as a 


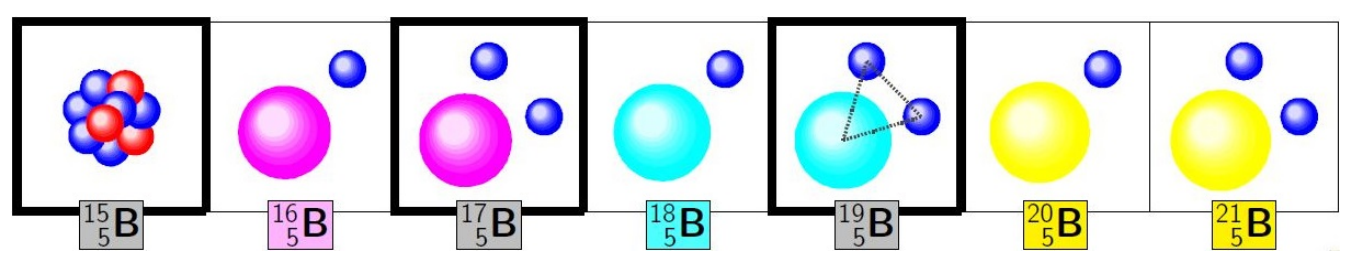

Figure 2: The boron chain from $A=15$ to 21 . The bound isotopes are marked within a thick frame, and the different number of clusters display the dominant structure of the corresponding ground states, from the 15 nucleons of ${ }^{15} \mathrm{~B}$ to the two- and three-body structures of all the heavier isotopes.

series of one neutron resonances on top of a core of 15 nucleons. By adding a second neutron, the system becomes bound again, although the two valence neutrons form a neutron halo around the same 15-nucleon core.

By adding an extra neutron, ${ }^{18} \mathrm{~B}$ is unbound again. However, the ground state is no longer a neutron resonance, but a virtual state with a huge scattering length of about $-100 \mathrm{fm}$ [8], the largest known $n$-nucleus scattering length. This surprisingly large value has been confirmed by the SAMURAI collaboration, and naturally opens the debate about the possibility of Efimov states or physics [11] in ${ }^{19} \mathrm{~B}$. The latter is a (weakly) bound system exhibiting a two-neutron halo, with a $2 n$ separation energy compatible with zero $\left(S_{2 n}=0.14 \pm 0.39 \mathrm{MeV}[12]\right)$. Note that it may also be considered as a four-neutron halo or skin, since as said above the ${ }^{17} \mathrm{~B}$ core is already a two-neutron halo itself (see Fig. 2).

The potential to exhibit Efimov physics has been investigated within a three-body model [13], in which ${ }^{19} \mathrm{~B}$ is built from potentials reproducing the known scattering lengths in the ${ }^{17} \mathrm{~B}+n(\sim-100 \mathrm{fm})$ and $n+n(\sim-20 \mathrm{fm})$ systems. With these simple ingredients, and without any three-body force, the model reproduces the basic features of the system within the experimental uncertainties: a weakly-bound ground state, with extended spatial distribution, and no bound excited states.

Surprisingly, these properties are even described at the unitary limit, in which both scattering lengths are taken to infinity. A more precise measurement of the key parameters, the ${ }^{19} \mathrm{~B}$ mass and the ${ }^{17} \mathrm{~B}+n$ scattering length, would provide a more stringent constraint on this description and on the Efimov character of the system.

Even this surprisingly complex and exotic system, ${ }^{19} \mathrm{~B}$, can play the role of a single body if we keep adding neutrons to the system. In fact, three $1 n$ resonances have been measured in ${ }^{20} \mathrm{~B}$, in which the exotic ${ }^{19} \mathrm{~B}$ structure acts like a core. By adding an extra neutron, a $2 n$ resonance has been observed in ${ }^{21} \mathrm{~B}$, again on top of a ${ }^{19} \mathrm{~B}$ core (Fig. 2).

Moreover, this resonance does not decay through the available ${ }^{20} \mathrm{~B}$ ones, but seems to emit the two neutrons directly in its decay towards ${ }^{19} \mathrm{~B}$, making of the ${ }^{21} \mathrm{~B}$ ground state a good candidate for the exotic $2 n$ emission.

This confirms the boron isotopes as an ideal ground for the study not only of exotic structures, but also of exotic decays.

\section{Z=4: 'dineutron' emission}

Concerning this exotic decay, the emission of a 'dineutron' was claimed from the interpretation of a low-energy $n-n$ signal in the decay of the unbound system ${ }^{16} \mathrm{Be}$ [14]. In fact, the signal was compared to the distributions from a free three-body ${ }^{14} \mathrm{Be}+n+n$ phase space and from a two-body ${ }^{14} \mathrm{Be}+{ }^{2} n$ phase space. Logically, the latter was closer to the data, and lead to the " $n$ emission" conclusion. However, a more realistic three-body phase space, taking into account 


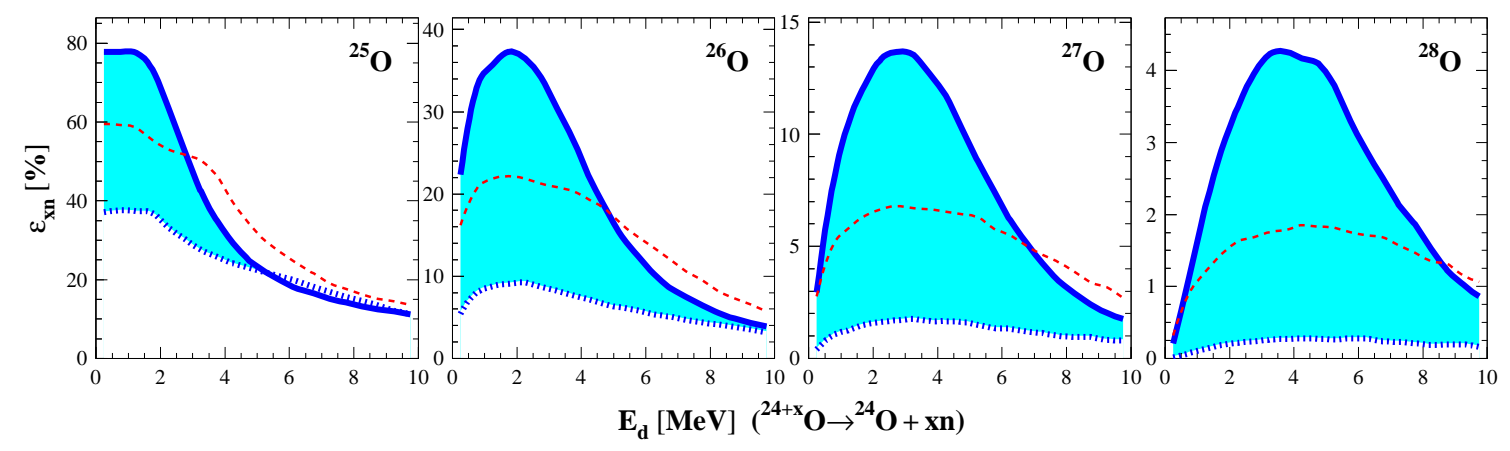

Figure 3: Simulated multi-neutron efficiency of the NEBULA array for the decay of unbound oxygen isotopes ${ }^{A} \mathrm{O}$ at $250 \mathrm{MeV}$ /nucleon into ${ }^{24} \mathrm{O}+(A-24) n$ as a function of the decay energy. The dotted blue line corresponds to the standard two-wall configuration of NEBULA, the solid blue line to the EXPAND extension to four walls, and the dashed red line to the addition of four double-planes of NeuLAND.

the attractive $n-n$ interaction, could also have described the experimental signal [15].

In order to shed light on this debate, a new experiment was performed by the SAMURAI collaboration using the reaction ${ }^{17} \mathrm{~B}(p, 2 p)^{14} \mathrm{Be}+n+n$. Thanks to the high luminosity and resolution provided by the MINOS target [16] and to the large acceptance of the neutron array NEBULA [17], in the range of the wide structure previously observed in Ref. [14] two narrow resonances were detected, identified with the $J^{\pi}=0^{+}$ground state and the $2^{+}$first excited state of ${ }^{16} \mathrm{Be}$.

The correlations in the decay of those two states show a direct $2 n$ emission (without going through any ${ }^{15} \mathrm{Be}$ resonance) with a very strong $n$ - $n$ signal, specially in the decay of the excited state. However, a 'dineutron' hypothesis is no longer needed to describe either of the two decays [18].

Work is in progress for the interpretation of the $n-n$ signal from microscopic principles. A three-body model of ${ }^{16} \mathrm{Be}$ from the ${ }^{14} \mathrm{Be}+n$ and $n+n$ potentials [19] describes well the two resonances observed. An extension of the model in order to calculate the $n-n$ signal that should be observed from the outgoing flux of the three-body wave function leads to good overall agreement for the ${ }^{16} \mathrm{Be}$ ground state.

More importantly, it links the origin of the signal with a compact $2 n$ lobe of the wave function, generated mainly by the $n-n$ interaction. Similar calculations are in progress for the $2^{+}$excited state, an even more challenging case.

\section{$4 \mathrm{Z}=1$ and 0: neutron matter}

The previous examples are definitely exotic, but correspond to $N / Z$ values ranging 'only' from 2 to 3 . In order to reach even larger asymmetries, one needs to go down in $Z$, as can be seen in Fig. 1. In the hydrogen chain, beyond the triton one finds wide resonances of $1,2,3 n$ respectively for ${ }^{4,5,6} \mathrm{H}$ in the range $2-3 \mathrm{MeV}$. However, ${ }^{7} \mathrm{H}$ has attracted special attention in recent decades. On one hand, its $N / Z=6$ is the closest one can get in the laboratory to the ratio found in neutron stars $(N / Z \sim 8)$. Therefore, it represents a unique benchmark for calculations of neutron-dominated matter.

On the other hand, the different experiments that have been undertaken (see for example Refs. $[20,21]$ ) point towards a very low-energy structure, at least below those of the preceding isotopes, which is extremely surprising for a system so far away from the dripline. 
However, all the different experiments had in common that the statistics and resolutions were low, the backgrounds important, and that the detection of ${ }^{7} \mathrm{H}$ was indirect (the missing mass of a binary reaction) due to the inability to detect the neutrons of the subsequent ${ }^{3} \mathrm{H}+4 n$ decay.

The landscape is in fact similar for the $Z=0$ case. After several decades of unsuccessful search for multi-neutron systems, the first positive (although weak) signal was observed at GANIL in the breakup of ${ }^{14} \mathrm{Be}$ into ${ }^{10} \mathrm{Be}+4 n$ [22]. However, several attempts were not able to confirm it, until an independent experiment at RIKEN, the double charge-exchange reaction ${ }^{4} \mathrm{He}\left({ }^{8} \mathrm{He},{ }^{8} \mathrm{Be}\right) 4 n$, found another positive but again weak signal [23].

In these cases as well, the detection of the tetraneutron was indirect.

In an attempt to answer these two fundamental questions, an experiment has been undertaken at RIKEN aiming to detect directly the formation of ${ }^{7} \mathrm{H}$ and its decay into $4 n$ [24]. In order to do so, the NEBULA neutron array was completed with a demonstrator of the NeuLAND array from GSI, allowing the detection of the four neutrons in coincidence with efficiencies higher than $1 \%$ (red line in Fig. 3).

The reaction ${ }^{8} \mathrm{He}(p, 2 p)\left\{{ }^{3} \mathrm{H}+4 n\right\}$ at $150 \mathrm{MeV} /$ nucleon on the MINOS target leads to a seven-body final state, with two protons at about $45^{\circ}$ and a triton and four neutrons at forward angles. The detection of all these particles will lead to an energy resolution for ${ }^{7} \mathrm{H}$ states of about $100 \mathrm{keV}$, an order of magnitude better than the previous missing-mass experiments.

Moreover, the detection of the full kinematics allows for the reconstruction of the $\left\{{ }^{3} \mathrm{H}+4 n\right\}$ system in its center of mass. If low-energy states of ${ }^{7} \mathrm{H}$ decay directly into ${ }^{3} \mathrm{H}$ (as expected due to the absence of low-energy resonances in ${ }^{4,5,6} \mathrm{H}$ ), the angular correlations in the decay will unambiguously show whether the phase space corresponds to two bodies instead of five, which would be the signature of the formation of a tetraneutron resonance in the decay.

\section{Conclusion}

During the recent SAMURAI campaigns at RIKEN many exotic nuclear structures have been (and are being) revealed. The neutron-rich end of the boron isotopic chain exhibits many of them, like two-neutron haloes and strong virtual states, the lighter ones being embedded into the heavier ones like a femtoscopic matryoshka.

At the end of this chain one finds ${ }^{21} \mathrm{~B}$, a candidate for $2 n$ emission. This exotic decay has been extensively studied in the case of ${ }^{16} \mathrm{Be}$, with the help of a microscopic three-body model that links the emission pattern to the structure of the wave function of the system.

Finally, the still open question about the existence of multi-neutron systems, like ${ }^{7} \mathrm{H}$ and the tetraneutron, has been addressed with a complete kinematics, high-resolution and highstatistics experiment. In the short-term future the results should bring definitive answers for both systems.

\section{Acknowledgements}

The results discussed in this contribution have been obtained by the SAMURAI collaboration, in particular the SAMURAI Day-One for the boron isotopes, SAMURAI- 18 for ${ }^{16} \mathrm{Be}$, and SAMURAI-34 for ${ }^{7} \mathrm{H}$ and the tetraneutron. 


\section{References}

[1] T. Nakamura, H.Sakurai and H.Watanabe, Exotic nuclei explored at in-flight separators, Prog. in Part. Nucl. Phys. 97, 53 (2017), doi:10.1016/j.ppnp.2017.05.001.

[2] M. Stanoiu et al., $N=14$ and 16 shell gaps in neutron-rich oxygen isotopes, Phys. Rev. C 69, 034312 (2004), doi:10.1103/PhysRevC.69.034312.

[3] C.R. Hoffman et al., Evidence for a doubly magic ${ }^{24}$ O, Phys. Lett. B 672, 17 (2009), doi:10.1016/j.physletb.2008.12.066.

[4] B. A. Brown, W. A. Richter, Magic numbers in the neutron-rich oxygen isotopes, Phys. Rev. C 72, 057301 (2005), doi:10.1103/PhysRevC.72.057301.

[5] T. Otsuka, T. Suzuki, J. D. Holt, A. Schwenk, and Y. Akaishi, Three-body forces and the limit of oxygen isotopes, Phys. Rev. Lett. 105, 032501 (2010), doi:10.1103/PhysRevLett.105.032501.

[6] T. Minamisono et al., Proton halo of ${ }^{8} B$ disclosed by its giant quadrupole moment, Phys. Rev. Lett. 69, 2058 (1992), doi:10.1103/PhysRevLett.69.2058.

[7] J. L. Lecouey et al., Single-proton removal reaction study of ${ }^{16} B$, Phys. Lett. B 672, 6 (2009), doi:10.1016/j.physletb.2008.12.053.

[8] A. Spyrou et al., First evidence for a virtual ${ }^{18}$ B ground state, Phys. Lett. B 683, 129 (2010), doi:10.1016/j.physletb.2009.12.016.

[9] T. Suzuki et al., Nuclear radii of ${ }^{17,19} \mathrm{~B}$ and ${ }^{14} \mathrm{Be}$, Nucl. Phys. A 658, 313 (1999), doi:10.1016/S0375-9474(99)00376-0.

[10] S. Leblond et al., First observation of ${ }^{20} B$ and ${ }^{21} B$, Phys. Rev. Lett. 121, 262502 (2018), doi:10.1103/PhysRevLett.121.262502.

[11] P. Naidon, S. Endo, Efimov physics: a review, Rep. Prog. Phys. 80, 5 (2017), doi:10.1088/1361-6633/aa50e8.

[12] L. Gaudefroy et al., Direct mass measurements of ${ }^{19} \mathrm{~B},{ }^{22} \mathrm{C},{ }^{29} \mathrm{~F},{ }^{31} \mathrm{Ne},{ }^{34} \mathrm{Na}$ and other light exotic nuclei, Phys. Rev. Lett. 109, 202503 (2012), doi:10.1103/PhysRevLett.109.202503.

[13] E. Hiyama, R. Lazauskas, F. M. Marqués, and J. Carbonell, Modeling ${ }^{19} B$ as a ${ }^{17} B-n-n$ three-body system in the unitary limit, Phys. Rev. C 100, 011603R (2019), doi:10.1103/PhysRevC.100.011603.

[14] A. Spyrou et al., First observation of ground state dineutron decay: ${ }^{16}$ Be, Phys. Rev. Lett. 108, 102501 (2012), doi:10.1103/PhysRevLett.108.102501.

[15] F. M. Marqués, N. A. Orr, N. L. Achouri, F. Delaunay, and J. Gibelin, Comment on "First Observation of Ground State Dineutron Decay: ${ }^{16}$ Be", Phys. Rev. Lett. 109, 239201 (2012), doi:10.1103/PhysRevLett.109.239201.

[16] A. Obertelli et al., MINOS: A vertex tracker coupled to a thick liquid-hydrogen target for inbeam spectroscopy of exotic nuclei, Eur. Phys. J. A 50, 8 (2014), doi:10.1140/epja/i201414008-y.

[17] T. Nakamura and Y. Kondo, Large acceptance spectrometers for invariant mass spectroscopy of exotic nuclei and future developments, Nucl. Instrum. Methods Phys. Res., Sect. B 376, 156 (2016), doi:10.1016/j.nimb.2016.01.003. 
[18] B. Monteagudo et al., in preparation.

[19] J. Casal, J. Gómez-Camacho, Identifying structures in the continuum: Application to ${ }^{16} \mathrm{Be}$, Phys. Rev. C 99, 014604 (2019), doi:10.1103/PhysRevC.99.014604.

[20] A. A. Korsheninnikov et al., Experimental evidence for the existence of ${ }^{7} \mathrm{H}$ and for a specific structure of ${ }^{8} \mathrm{He}$, Phys. Rev. Lett. 90, 082501 (2003), doi:10.1103/PhysRevLett.90.082501.

[21] M. Caamaño et al., Resonance State in ${ }^{7} H$, Phys. Rev. Lett. 99, 062502 (2007), doi:10.1103/PhysRevLett.99.062502.

[22] F.M. Marqués et al., Detection of neutron clusters, Phys. Rev. C 65, 044006 (2002), doi:10.1103/PhysRevC.65.044006.

[23] K. Kisamori et al., Candidate resonant tetraneutron state populated by the ${ }^{4} \mathrm{He}\left({ }^{8} \mathrm{He},{ }^{8} \mathrm{Be}\right)$ reaction, Phys. Rev. Lett. 116, 052501 (2016), doi:10.1103/PhysRevLett.116.052501.

[24] F.M. Marqués et al., RIBF Experimental Proposal NP1512-SAMURAI34 (2015). 